\title{
Coherent Steps of Mobile Sensing Agents in Gaussian Scalar Fields
}

\author{
Wencen $\mathrm{Wu}$ and Fumin Zhang
}

\begin{abstract}
This paper develops fundamental theoretical results to describe exploration behaviors of mobile sensing agents in a noisy scalar field. We introduce concepts of coherent steps and incoherent steps of the sensing agents and prove that a step of an agent is coherent if and only if the probability of a false-walk is less than a threshold determined by the explorable probability. Among all possible coherent steps, gradient climbing and level curve tracking are the steps that can achieve local supremum explorable probabilities. We also prove that by increasing the number of agents that are collaborating, the incoherent steps of the collaborating groups can become coherent. Based on estimates of the noise variance, we propose an algorithm that estimates the minimum number of agents required to guarantee coherent steps and implement a strategy that allows the sensing agents to self-organize into groups with the estimated minimum number of agents. Results are demonstrated in multi-robot experiments.
\end{abstract}

\section{INTRODUCTION}

An important application for mobile robotics is to deploy mobile robots to explore unknown scalar fields (e.g., chemical fields, temperature fields, etc). Various exploration missions such as gradient climbing/descending [1]-[5] and level curve tracking [6]-[9] have been investigated in the literature. Some work focuses on developing exploration strategies using one agent [10]-[14]. However, due to the advantages of cooperative exploration over individual exploration in certain aspects such as adaptiveness and efficiency, recent work has focused on using multiple agents [8], [15][18]. Various algorithms have been developed or extended to control a group of agents that better perform exploration tasks.

Our previous work [19] investigates how difficult exploring a field is with a certain amount of agents. We introduce concepts of local explorable fields and false-walks of sensing agents, and provide criteria such as the explorable probability of a location in a scalar field and the probability of a falsewalk of a sensing agent moving in the field. In this paper, we focus on the behaviors of sensing agents in the exploration of unknown noisy scalar fields based on explorability analysis. We improve the definition of local explorability, and introduce notions of coherent steps and incoherent steps according to the movements of the agents and field values along the trajectories of the agents. We investigate the relationships between local explorable fields and coherent steps of sensing agents, as well as the relationships between

The research work is supported by ONR grants N00014-08-1-1007, N00014-09-1-1074, and N00014-10-10712 (YIP), and NSF grants ECCS0841195 (CAREER), CNS-0931576, and ECCS-1056253. Wencen Wu and Fumin Zhang are with the School of Electrical and Computer Engineering, Georgia Institute of Technology, Atlanta, GA, 30332, USA wwencen3, fuminegatech.edu coherent steps and the probability of false-walks. We prove that, a step being coherent is the sufficient and necessary condition for the probability of a false-walk being less than a threshold determined by the explorable probability. Therefore, coherent steps guarantee lower probability of a false-walk compared to incoherent steps. We also show that, among all coherent steps, gradient climbing/descending and level curve tracking are two of the steps that can achieve local supremum explorable probabilities.

Since incoherent steps are associated with higher probabilities of false-walks, we wish to avoid incoherent steps. One method is to perform cooperative exploration that uses multiple agents and generates filtered measurements. We apply the cooperative exploration strategy introduced in [8] and find that by increasing the number of agents that are collaborating, the steps of the group of agents can become coherent, which guarantees a low probability of false-walks. However, the more collaborating agents, the higher the communication and computation costs. Therefore, we wish to minimize the number of agents to guarantee coherent steps. We design an estimating algorithm to estimate the minimum number of agents and apply the algorithm to experiments that include several Khepera III robots deployed in a light field seeking a light source. When the robots decide that they need to collaborate, we allow the robots to self-organize into groups with the estimated minimum number of robots. This estimation can also be adopted in various problems such as self-organization, task allocation, and explore vs. exploit problem when a certain number of agents need to be assigned to new tasks. Several studies regarding self-organizing swarms [20]-[22] develop procedures of organization, while our results provide an estimate of the number of agents required to guarantee coherent steps.

The rest of the paper is organized as follows. Section II introduces the definition of local explorability of a scalar field and false-walks of sensing agents. Section III proposes the concepts of coherent steps and incoherent steps, and investigates the relationships between the coherent steps and probability of false-walks. Section IV examines cooperative exploration based on explorability analysis. Section V proposes an algorithm that estimates the minimum number of agents that are required to guarantee coherent steps and provides experimental results. Section VI presents concluding remarks.

\section{EXPLORABILITY OF NOISY SCALAR FIELDS}

Consider a noisy scalar field $Y(\mathbf{x})=F(\mathbf{x})+W(\mathbf{x})$, in which $\mathbf{x} \in \mathbb{R}^{n}$ denotes a location in the field, $F(\mathbf{x})=E(Y(\mathbf{x}))$ the smooth mean field, and $W(\mathbf{x})$ the noise. In this paper, 
for $Y(\mathbf{x})$ and $W(\mathbf{x})$, we use upper case letters to denote random variables and lower case letters to denote values of random variables. $E(\cdot)$ represents expectations. Suppose $\mathbf{x}_{0} \in \mathbb{R}^{n}$ is a regular point of $F(\mathbf{x})$ that indicates $d F\left(\mathbf{x}_{0}\right) \neq 0$, e.g., $\nabla F\left(\mathbf{x}_{0}\right) \neq 0$. Define sets $U^{+}(\varepsilon)=\left\{\mathbf{x} \mid F(\mathbf{x})-F\left(\mathbf{x}_{0}\right)>\right.$ $\varepsilon\}, U^{-}(\varepsilon)=\left\{\mathbf{x} \mid F(\mathbf{x})-F\left(\mathbf{x}_{0}\right)<-\varepsilon\right\}, U^{0}(\varepsilon)=\{\mathbf{x}|| F(\mathbf{x})-$ $\left.F\left(\mathbf{x}_{0}\right) \mid \leq \varepsilon\right\}$, and $B\left(\delta_{1}\right)=\left\{\mathbf{x} \mid\left\|\mathbf{x}-\mathbf{x}_{0}\right\| \leq \delta_{1}\right\}$, where $\varepsilon>0$ and $\delta_{1}>0$ are two constants. We define local explorability as follows.

Definition 2.1: Given $\varepsilon>0$ and $0<p<1$, field $Y(\mathbf{x})$ is locally $(p, \varepsilon)$ explorable at $\mathbf{x}_{0}$ (i.e., $\mathbf{x}_{0}$ is locally $(p, \varepsilon)$ explorable) if there exist $\varepsilon^{+}>\varepsilon$ and $0<\varepsilon^{0}<\varepsilon$, such that for $\forall \delta_{1}>0$ satisfying that $\left(U^{+}\left(\varepsilon^{+}\right) \cup U^{-}\left(\varepsilon^{+}\right) \cup U^{0}\left(\varepsilon^{0}\right)\right) \cap B\left(\delta_{1}\right)$ has a non-zero Lebesgue measure, the following conditions are satisfied:

1. If $\mathbf{x} \in U^{+}\left(\varepsilon^{+}\right) \cap B\left(\delta_{1}\right), \operatorname{Pr}\left(Y(\mathbf{x})>Y\left(\mathbf{x}_{0}\right)+\varepsilon\right)>\frac{1+p}{2}$.

2. If $\mathbf{x} \in U^{-}\left(\varepsilon^{+}\right) \cap B\left(\delta_{1}\right), \operatorname{Pr}\left(Y(\mathbf{x})<Y\left(\mathbf{x}_{0}\right)-\varepsilon\right)>\frac{1+p}{2}$.

3. If $\mathbf{x} \in U^{0}\left(\varepsilon^{0}\right) \cap B\left(\delta_{1}\right), \operatorname{Pr}\left(\left|Y(\mathbf{x})-Y\left(\mathbf{x}_{0}\right)\right| \leq \varepsilon\right)>\frac{1+p}{2}$.

Moreover, $Y(\mathbf{x})$ is $(p, \varepsilon)$ explorable on an open set $C(\mathbf{x})$, in which measurements are taken if for every $\mathbf{x}_{0} \in C(\mathbf{x}), Y(\mathbf{x})$ is locally $(p, \varepsilon)$ explorable at $\mathbf{x}_{0}$. We refer to $p$ as the explorable probability and $\varepsilon$ as the resolution. $\delta_{1}$ is the distance between positions $\mathbf{x}_{0}$ and $\mathbf{x}$.

When exploring a field using mobile sensing agents, we evaluate the difference in field values through measurements of the agents. Suppose an agent is discretely taking measurements of field $Y(\mathbf{x})$ while moving in the field. We assume that each agent takes one measurement at each time instant. To describe the movement, we first define a step of an agent.

Definition 2.2: A step is a movement performed by a sensing agent from $\mathbf{x}_{0}$ to $\mathbf{x}$ in field $Y(\mathbf{x})$ with step size $\delta_{1}$, which satisfies $\left(U^{+}(\varepsilon) \cup U^{-}(\varepsilon)\right) \cap B\left(\delta_{1}\right) \neq \emptyset$.

At locations $\mathbf{x}_{0}$ and $\mathbf{x}$, the agent takes two measurements, $y\left(\mathbf{x}_{0}\right)$ and $y(\mathbf{x})$, respectively. Based on the direction of movement from $\mathbf{x}_{0}$ to $\mathbf{x}$, field value $F(\mathbf{x})$ may increase or decrease, or remain unchanged compared to $F\left(\mathbf{x}_{0}\right)$. However, due to the noises, the difference between $y(\mathbf{x})$ and $\mathbf{y}\left(\mathbf{x}_{0}\right)$ may not be consistent with the difference between $F(\mathbf{x})$ and $F\left(\mathbf{x}_{0}\right)$. To evaluate the inconsistence, we introduce the following concept of false-walks [19].

Definition 2.3: A false-walk is a step performed by a sensing agent such that one of the following conditions is satisfied:

1. If $\mathbf{x} \in U^{+}(\varepsilon)$, then $y(\mathbf{x}) \leq y\left(\mathbf{x}_{0}\right)+\varepsilon$.

2. If $\mathbf{x} \in U^{-}(\varepsilon)$, then $y(\mathbf{x}) \geq y\left(\mathbf{x}_{0}\right)-\varepsilon$.

3. If $\mathbf{x} \in U^{0}(\varepsilon)$, then $\left|y(\mathbf{x})-y\left(\mathbf{x}_{0}\right)\right|>\varepsilon$.

A false-walk indicates the inconsistence in the measurements of an agent and the mean field values, which causes inaccurate extraction of information from the sensor readings. Thus, in desirable exploration tasks, we want to reduce the probability of false-walks.

We introduce a proposition regarding the relationships between false-walks and local explorability. Define the set $\Omega=U^{+}\left(\varepsilon^{+}\right) \cup U^{-}\left(\varepsilon^{+}\right) \cup U^{0}\left(\varepsilon^{0}\right)$. The proposition is as follows.

Proposition 2.4: Given $\varepsilon>0$ and $0<p<1$, field $Y(\mathbf{x})$ is locally $(p, \varepsilon)$ explorable at location $\mathbf{x}_{0}$ if and only if there exist $\varepsilon^{+}>\varepsilon$ and $0<\varepsilon^{0}<\varepsilon$, such that for $\forall \delta_{1}>0$ satisfying that $\Omega \cap B\left(\delta_{1}\right)$ has a non-zero Lebesgue measure, the probability of a false-walk performed by a sensing agent from $\mathbf{x}_{0}$ to $\mathbf{x} \in \Omega$ with step size $\delta_{1}$ satisfies $\operatorname{Pr}(F W) \leq \frac{1-p}{2}$.

The proof of the proposition is straightforward from the definition of the local explorability. The proposition implies that the explorable probability $p$ is an indictor of the probability of a false-walk. We consider the explorable probability as one criterion of evaluating the difficulty of exploring a field.

\section{COHERENT STEPS AND INCOHERENT STEPS}

When an agent is moving in field $Y(\mathbf{x})$, we need to determine which steps can guarantee a low probability of false-walks. To address this task, we introduce the concepts of coherent steps and incoherent steps of an agent in this section and discuss the relationships between those steps and the probability of false-walks. For sensor measurements and field noises, we assume that the following assumption holds for field $Y(\mathbf{x})$ throughout the paper.

Assumption 3.1: Field noise $W(\mathbf{x})$ is independent and identically distributed (i.i.d.) Gaussian with zero mean and variance $\sigma^{2}$.

We assume the measurement noise to be zero-mean Gaussian and consider it together with the field noise. Therefore, the measurement taken by the agent at $t_{k}$ can be written as

$$
y\left(\mathbf{x}_{k}\right)=F\left(\mathbf{x}_{k}\right)+w\left(\mathbf{x}_{k}\right),
$$

in which the noise term $w\left(\mathbf{x}_{k}\right)$ can be considered as consisting of both field noise and measurement noise.

Suppose a sensing agent is moving in field $Y(\mathbf{x})=F(\mathbf{x})+$ $W(\mathbf{x})$ under Assumption 3.1. The direction of the movement depends on different tasks such as gradient climbing and level curve tracking. Based on the movement of the agent, we define coherent steps of an agent as follows.

Definition 3.2: Given a noisy Gaussian scalar field $Y(\mathbf{x})=$ $F(\mathbf{x})+W(\mathbf{x})$ and two constants $\varepsilon>0$ and $0<p<1$, a step of an agent from $\mathbf{x}_{0}$ to $\mathbf{x}$ with a step size $\delta_{1}$ is coherent if one of the following conditions is satisfied.

(1) When $\mathbf{x} \in U^{+}(\varepsilon) \cap B\left(\delta_{1}\right)$ or $\mathbf{x} \in U^{-}(\varepsilon) \cap B\left(\delta_{1}\right)$,

$$
\frac{\left|F(\mathbf{x})-F\left(\mathbf{x}_{0}\right)\right|-\varepsilon}{\sigma}>2 \operatorname{erf}^{-1}(p) \text {. }
$$

(2) When $\mathbf{x} \in U^{0}(\varepsilon) \cap B\left(\delta_{1}\right)$,

$\operatorname{erf}\left(\frac{\varepsilon-\left(F(\mathbf{x})-F\left(\mathbf{x}_{0}\right)\right)}{2 \sigma}\right)+\operatorname{erf}\left(\frac{\varepsilon+\left(F(\mathbf{x})-F\left(\mathbf{x}_{0}\right)\right)}{2 \sigma}\right)>1+p$.

Otherwise, the step of the agent is incoherent.

In the definition, erf represents the Gauss error function. Given the definition, we have the following propositions regarding the local explorability and coherent steps.

Proposition 3.3: Given $\varepsilon>0$ and $0<p<1$, field $Y(\mathbf{x})$ with Gaussian noise $W(\mathbf{x})$ is locally $(p, \varepsilon)$ explorable at location $\mathbf{x}_{0}$ if and only if there exist $\varepsilon^{+}>\varepsilon$ and $0<\varepsilon^{0}<\varepsilon$, such that for $\forall \delta_{1}>0$ satisfying that $\Omega \cap B\left(\delta_{1}\right)$ has a non-zero Lebesgue measure, any step of the agent from $\mathbf{x}_{0}$ to $\mathbf{x} \in \Omega$ with a step size $\delta_{1}$ is coherent. 
Proof: We first prove the necessary condition. Given two locations $\mathbf{x}_{0}$ and $\mathbf{x}$, the agent measures two random variables, $Y\left(\mathbf{x}_{0}\right)$ and $Y(\mathbf{x})$. We define a new random variable $Z(\mathbf{x})$ that satisfies $Z(\mathbf{x})=W(\mathbf{x})-W\left(\mathbf{x}_{0}\right)$. Then, we obtain $Y(\mathbf{x})-Y\left(\mathbf{x}_{0}\right)=F(\mathbf{x})-F\left(\mathbf{x}_{0}\right)+Z(\mathbf{x})$. Based on the definition of local explorability, the following cases are discussed.

(1) When $\mathbf{x} \in U^{+}\left(\varepsilon^{+}\right) \cap B\left(\delta_{1}\right)$, which indicates $F(\mathbf{x})-$ $F\left(\mathbf{x}_{0}\right)>\varepsilon^{+}$, we calculate $\operatorname{Pr}\left(Y(\mathbf{x})>Y\left(\mathbf{x}_{0}\right)+\varepsilon\right)=\operatorname{Pr}(Z(\mathbf{x})>$ $\left.\varepsilon-\left(F(\mathbf{x})-F\left(\mathbf{x}_{0}\right)\right)\right)=1-\int_{-\infty}^{\varepsilon-\left(F(\mathbf{x})-F\left(\mathbf{x}_{0}\right)\right)} f(z) d z>\frac{1+p}{2}$, where $f(z)$ is the probability density function (p.d.f.) of the random variable $Z(\mathbf{x})$. Since we assume that noise $W(\mathbf{x})$ is i.i.d. Gaussian with variance $\sigma^{2}$ and zero mean, then $Z(\mathbf{x})$ is also i.i.d. Gaussian with variance $2 \sigma^{2}$ and zero mean. Therefore, we obtain $\operatorname{Pr}\left(Y(\mathbf{x})>Y\left(\mathbf{x}_{0}\right)+\varepsilon\right)=1-$ $\frac{1}{\sqrt{4 \pi \sigma^{2}}} \int_{-\infty}^{\varepsilon-\left(F(\mathbf{x})-F\left(\mathbf{x}_{0}\right)\right)} e^{-\frac{z^{2}}{4 \sigma^{2}}} d z=\frac{1}{2}\left(1+\operatorname{erf}\left(\frac{\left(F(\mathbf{x})-F\left(\mathbf{x}_{0}\right)\right)-\varepsilon}{2 \sigma}\right)\right)>$ $\frac{1+p}{2}$, which yields $\frac{\left(F(\mathbf{x})-F\left(\mathbf{x}_{0}\right)\right)-\varepsilon}{\sigma}>2 \operatorname{erf}^{-1}(p)$.

(2) When $\left.\mathbf{x} \in U^{-}{ }^{\sigma} \varepsilon^{+}\right) \cap B\left(\delta_{1}\right)$, which indicates $F(\mathbf{x})-F\left(\mathbf{x}_{0}\right)<-\varepsilon^{+}$, we derive $\operatorname{Pr}\left(Y(\mathbf{x})<Y\left(\mathbf{x}_{0}\right)-\varepsilon\right)=$ $\int_{-\infty}^{-\varepsilon-\left(F(\mathbf{x})-F\left(\mathbf{x}_{0}\right)\right)} f(z) d z=\frac{1}{2}\left(1+\operatorname{erf}\left(\frac{-\varepsilon-\left(F(\mathbf{x})-F\left(\mathbf{x}_{0}\right)\right)}{2 \sigma}\right)\right)>\frac{1+p}{2}$. Then we obtain $\frac{-\left(F(\mathbf{x})-F\left(\mathbf{x}_{0}\right)\right)-\varepsilon}{\sigma}>2 \operatorname{erf}^{-1}(p)$. We conclude from the above two cases that

$$
\frac{\left|F(\mathbf{x})-F\left(\mathbf{x}_{0}\right)\right|-\varepsilon}{\sigma}>2 \operatorname{erf}^{-1}(p),
$$

which satisfies Equation (2) in Definition 3.2.

(3) When $\mathbf{x} \in U^{0}\left(\varepsilon^{+}\right) \cap B\left(\delta_{1}\right)$, which indicates $\left|F(\mathbf{x})-F\left(\mathbf{x}_{0}\right)\right| \leq \varepsilon^{+}$, we obtain $\operatorname{Pr}\left(\left|Y(\mathbf{x})-Y\left(\mathbf{x}_{0}\right)\right| \leq\right.$ $\varepsilon)=\int_{-\varepsilon-\left(F(\mathbf{x})-F\left(\mathbf{x}_{0}\right)\right)}^{\varepsilon-\left(F(\mathbf{x})-F\left(\mathbf{x}_{0}\right)\right)} f(z) d z=\frac{1}{2}\left(\operatorname{erf}\left(\frac{\varepsilon-\left(F(\mathbf{x})-F\left(\mathbf{x}_{0}\right)\right)}{2 \sigma}\right)+\right.$ $\left.\operatorname{erf}\left(\frac{\varepsilon+\left(F(\mathbf{x})-F\left(\mathbf{x}_{0}\right)\right)}{2 \sigma}\right)\right)>\frac{1+p}{2}$. Then, we derive $\operatorname{erf}\left(\frac{\varepsilon-\left(F(\mathbf{x})-F\left(\mathbf{x}_{0}\right)\right)}{2 \sigma}\right)+\operatorname{erf}\left(\frac{\varepsilon+\left(F(\mathbf{x})-F\left(\mathbf{x}_{0}\right)\right)}{2 \sigma}\right)>1+p$, which satisfies Equation (3) in Definition 3.2. The above derivations imply that for a field that is locally $(p, \varepsilon)$ explorable, if an agent moves from $\mathbf{x}_{0}$ to $\mathbf{x}$ with step size $\delta_{1}$, which satisfies $\mathbf{x} \in \Omega$, the step is coherent.

For the sufficient condition, since any step from $\mathbf{x}_{0}$ to $\mathbf{x} \in \Omega$ is coherent, then if $\mathbf{x} \in U^{+}\left(\varepsilon^{+}\right) \cap B\left(\delta_{1}\right)$ or $\mathbf{x} \in U^{-}\left(\varepsilon^{+}\right) \cap B\left(\delta_{1}\right)$, Equation (2) holds. From Equation (2), we derive $\operatorname{Pr}\left(Y(\mathbf{x})>Y\left(\mathbf{x}_{0}\right)+\varepsilon\right)>\frac{1+p}{2}$ or $\operatorname{Pr}\left(Y(\mathbf{x})<Y\left(\mathbf{x}_{0}\right)-\right.$ $\varepsilon)>\frac{1+p}{2}$. Similarly, from Equation (3), we derive $\operatorname{Pr}(\mid Y(\mathbf{x})-$ $\left.Y\left(\mathbf{x}_{0}\right) \mid \leq \varepsilon\right)>\frac{1+p}{2}$. Therefore, Definition 2.1 is satisfied, indicating that the field is $(p, \varepsilon)$ explorable.

Proposition 3.3 implies the following two corollaries.

Corollary 3.4: The probability of a false-walk from $\mathbf{x}_{0}$ to $\mathbf{x} \in \Omega$ satisfies $\operatorname{Pr}(F W) \leq \frac{1-p}{2}$ if and only if the step is coherent.

Corollary 3.5: Given $\varepsilon>0$ and $0<p<1$, a step from $\mathbf{x}_{0}$ to $\mathbf{x} \in \Omega$ with step size $\delta_{1}$ is coherent if there exist $\varepsilon^{+}>\varepsilon$ and $0<\varepsilon^{0}<\varepsilon$, such that for $\forall \delta_{1}>0$ satisfying that $\Omega \cap B\left(\delta_{1}\right)$ has a non-zero Lebesgue measure, the noise variance $\sigma^{2}$ satisfies one of the following conditions:

1. If $\mathbf{x} \in U^{+}\left(\varepsilon^{+}\right) \cap B\left(\delta_{1}\right)$ or $\mathbf{x} \in U^{-}\left(\varepsilon^{+}\right) \cap B\left(\delta_{1}\right), \sigma<$ $\frac{\varepsilon^{+}-\varepsilon}{2 e r f^{-1}(p)}$

2. If $\mathbf{x} \in U^{0}\left(\varepsilon^{0}\right) \cap B\left(\delta_{1}\right), \operatorname{erf}\left(\frac{\varepsilon-\varepsilon^{0}}{2 \sigma}\right)+\operatorname{erf}\left(\frac{\varepsilon+\varepsilon^{0}}{2 \sigma}\right)>1+p$. Corollary 3.4 suggests that compared to incoherent steps, coherent steps imply a lower probability of false-walks.
The probability of false-walks decreases as the explorable probability $p$ increases. Corollary 3.5 implies that as long as the noise variance satisfies one of the conditions in Corollary 3.5, the probability of a false-walk from $\mathbf{x}_{0}$ to $\mathbf{x} \in \Omega$ satisfies $\operatorname{Pr}(F W) \leq \frac{1-p}{2}$.

We have seen from Theorem 3.3 that, for any given field that is locally $(p, \varepsilon)$ explorable at $\mathbf{x}_{0}$, as long as $\mathbf{x} \in \Omega$, the step of an agent from $\mathbf{x}_{0}$ to $\mathbf{x}$ is coherent. In real-world applications, the step size or the speed of a robot and the resolution of a sensor are usually pre-determined and fixed. Therefore, given any fixed step size $\delta_{1}$ and resolution $\varepsilon$, we aim at finding the steps that can achieve maximum $p$. To examine this problem, we first define gradient climbing/descending and level curve tracking as follows.

Definition 3.6: Given scalar field $F(\mathbf{x})$ and an agent that moves one step from $\mathbf{x}_{0}$ to $\mathbf{x}$ with step size $\sigma_{1}$ in the field, if $\mathbf{x}-\mathbf{x}_{0}= \pm \delta_{1} \frac{\nabla F\left(\mathbf{x}_{0}\right)}{\left|\nabla F\left(\mathbf{x}_{0}\right)\right|}$, where $\nabla F\left(\mathbf{x}_{0}\right)$ is the gradient of the field at location $\mathbf{x}_{0}$, the agent is climbing/descending the gradient. If $F(\mathbf{x})-F\left(\mathbf{x}_{0}\right)=0$, the agent is tracking level curves.

Based on the definition, we introduce the following proposition.

Proposition 3.7: For an agent that moves one step from $\mathbf{x}_{0}$ to $\mathbf{x} \in \Omega$ with step size $\delta_{1}$ in field $Y(\mathbf{x})$, which is locally $(p, \varepsilon)$ explorable at $\mathbf{x}_{0}$, gradient climbing/descending and level curve tracking are the steps that can obtain local supremum explorable probabilities among all possible directions $\frac{\mathbf{x}-\mathbf{x}_{0}}{\left\|\mathbf{x}-\mathbf{x}_{0}\right\|}$.

Proof: Based on the location of $\mathbf{x}$, two situations occur.

(1) When $\quad \mathbf{x} \in U^{+}\left(\varepsilon^{+}\right) \cap B\left(\delta_{1}\right) \quad$ or $\quad \mathbf{x} \in U^{-}\left(\varepsilon^{+}\right) \cap B\left(\delta_{1}\right)$, $\left|F(\mathbf{x})-F\left(\mathbf{x}_{0}\right)\right|>\varepsilon$ and Equation (2) should be satisfied. Since the error function $\operatorname{erf}(\cdot)$ is monotonically increasing, we obtain $p<\operatorname{erf}\left(\frac{\left|F(\mathbf{x})-F\left(\mathbf{x}_{0}\right)\right|-\varepsilon}{2 \sigma}\right)$. Since $p$ can be arbitrarily close to $\operatorname{erf}\left(\frac{\left|F(\mathbf{x})-F\left(\mathbf{x}_{0}\right)\right|-\varepsilon}{2 \sigma}\right)$, then, when $\frac{\left|F(\mathbf{x})-F\left(\mathbf{x}_{0}\right)\right|-\varepsilon}{\sigma}$ increases, the upper bound of $p$ increases as well. Thus, for any fixed $\varepsilon$, when $\mathbf{x}-\mathbf{x}_{0}$ is aligned with the gradient direction, e.g. in linear case, $\mathbf{x}-\mathbf{x}_{0}= \pm \delta_{1} \frac{\nabla F\left(\mathbf{x}_{0}\right)}{\left\|\nabla F\left(\mathbf{x}_{0}\right)\right\|}$ and $\left|F(\mathbf{x})-F\left(\mathbf{x}_{0}\right)\right|=\delta_{1}\left\|\nabla F\left(\mathbf{x}_{0}\right)\right\|$, we can obtain the supremum of $p$ as $p_{\text {sup }}=\operatorname{erf}\left(\frac{\delta_{1}\left\|\nabla F\left(\mathbf{x}_{0}\right)\right\|-\varepsilon}{2 \sigma}\right)$.

(2) When $\mathbf{x} \in U^{0}\left(\varepsilon^{0}\right) \cap B\left(\delta_{1}\right)$, Equation (3) needs to be satisfied. We derive three special cases from Equation (3).

1) $F(\mathbf{x})-F\left(\mathbf{x}_{0}\right)= \pm \varepsilon^{0}$. In this case, Equation (3) becomes $\operatorname{erf}\left(\frac{\varepsilon-\varepsilon^{0}}{2 \sigma}\right)+\operatorname{erf}\left(\frac{\varepsilon+\varepsilon^{0}}{2 \sigma}\right)>1+p$

2) $F(\mathbf{x})-F\left(\mathbf{x}_{0}\right)=0$. In this case, Equation (3) becomes $2 \operatorname{erf}\left(\frac{\varepsilon}{2 \sigma}\right)>1+p$

3) When $F(\mathbf{x})-F\left(\mathbf{x}_{0}\right)$ goes from $-\varepsilon^{0}$ to 0 , the left side of Equation (3) increases from $\operatorname{erf}\left(\frac{\varepsilon-\varepsilon^{0}}{2 \sigma}\right)+\operatorname{erf}\left(\frac{\varepsilon+\varepsilon^{0}}{2 \sigma}\right)$ to $2 \operatorname{erf}\left(\frac{\varepsilon}{2 \sigma}\right)$; and when $F(\mathbf{x})-F\left(\mathbf{x}_{0}\right)$ goes from 0 to $\varepsilon^{0}$, the left side of the Equation (3) decreases from $2 \operatorname{erf}\left(\frac{\varepsilon}{2 \sigma}\right)$ to $\operatorname{erf}\left(\frac{\varepsilon-\varepsilon^{0}}{2 \sigma}\right)+\operatorname{erf}\left(\frac{\varepsilon+\varepsilon^{0}}{2 \sigma}\right) . p$ can also be arbitrarily close to $2 \operatorname{erf}\left(\frac{\varepsilon}{2 \sigma}\right)-1$. Therefore, the local supremum of $p$ is $p_{\text {sup }}=2 \operatorname{erf}\left(\frac{\varepsilon}{2 \sigma}\right)-1$.

From the above discusstion, when the moving direction is aligned with gradient directions or level curves, supremum explorable probability can be achieved. 
Note that, incoherent steps occur when an agent moves one step from $\mathbf{x}_{0}$ to any location $\mathbf{x}$ with step size $\delta_{1}$ in field $Y(\mathbf{x})$ that is not locally $(p, \varepsilon)$ explorable at $\mathbf{x}_{0}$, or from $\mathbf{x}_{0}$ to $\mathbf{x}$ that does not belong to set $\Omega$ with step size $\delta_{1}$ in field $Y(\mathbf{x})$ that is locally $(p, \varepsilon)$ explorable at $\mathbf{x}_{0}$. From Corollary 3.5 , we wish to control the agents to perform coherent steps to guarantee low probability of false-walks.

\section{CoOperative Exploration in Gaussian Fields}

Corollary 3.5 implies that for fixed locations $\mathbf{x}_{0}$ and $\mathbf{x}$ and fixed constants $p$ and $\varepsilon$, noise variance $\sigma^{2}$ is the factor that determines whether a step of an agent is coherent or not. Since the noise term consists of both field noise and measurement noise, we investigate cooperative exploration using multiple sensing agents that the measurement noise can be filtered by combining measurements from multiple agents.

Suppose we deploy $N$ mobile sensing agents in field $Y(\mathbf{x})$. Denote the position of the $i$ th agent at the $k$ th time step as $\mathbf{x}_{i, k}, i=1, \cdots, N$ and the measurement taken by the agent as $y\left(\mathbf{x}_{i, k}\right)$. When the $N$ agents are collaborating, we assume that they move in a constant formation. In this paper, formation control is achieved by using Jacobi transform based method [8], [23], [24], which decouples the dynamics of the formation from the motion of the center of the formation. Therefore, separate control laws can be designed for formation control and motion control. As the agents are collaborating, we treat them as a group and refer to the group as a "super-agent", whose position is denoted by the center of the formation that satisfies $\mathbf{x}_{c, k}=\frac{1}{N} \sum \mathbf{x}_{i, k}$.

To obtain filtered measurements from the measurements of the $N$ collaborating agents, we construct a cooperative Kalman filter, which produces estimated field values and gradients at the center of the formation. We choose the state to be $\mathbf{s}_{k}=\left(F\left(\mathbf{x}_{c, k}\right), \nabla F\left(\mathbf{x}_{c, k}\right)^{T}\right)^{T}$, in which $\nabla F\left(\mathbf{x}_{c, k}\right)^{T}$ is the gradient at the center of the formation. Define $A_{k-1}=\left(\begin{array}{cc}1 & \left(\mathbf{x}_{c, k}-\mathbf{x}_{c, k-1}\right)^{T} \\ 0 & \mathbf{I}_{2 \times 2}\end{array}\right)$ and $\mathbf{h}_{k-1}=$ $\left(0, E\left[H_{c, k-1}\left(\mathbf{x}_{c, k}-\mathbf{x}_{c, k-1}\right)\right]^{T}\right)^{T}$, where $H_{c, k-1}$ is the estimate of the Hessian at the center of the formation. Let $C_{k}$ be the $N \times 3$ matrix with its $i$ th row defined by $\left[1,\left(\mathbf{x}_{i, k}-\mathbf{x}_{c, k}\right)^{T}\right]$ for $i=1,2, \ldots, N$ and $D_{k}$ the $N \times 4$ matrix with its $i$ th row vector defined by the Kronecker product $\frac{1}{2}\left(\left(\mathbf{x}_{i, k}-\mathbf{x}_{c, k}\right) \otimes\right.$ $\left.\left(\mathbf{x}_{i, k}-\mathbf{x}_{c, k}\right)\right)^{T}$. We define the $N \times 1$ measurement vector $\mathbf{p}_{k}=\left[y\left(\mathbf{x}_{i, k}\right)\right]$ and write down the state equation and the measurement equation as follows

$$
\begin{aligned}
& \mathbf{s}_{k}=A_{k-1} \mathbf{s}_{k-1}+\mathbf{h}_{k-1}+\mathbf{v}_{k-1}, \\
& \mathbf{p}_{k}=C_{k} \mathbf{s}_{k}+D_{k} \vec{H}_{c, k}+\mathbf{w}_{k},
\end{aligned}
$$

where $\mathbf{v}_{k-1}$ is the $3 \times 1$ modeling noise vector, which accounts for positioning errors, estimation errors for the Hessians, and errors caused by higher-order terms omitted from the Taylor expansion. $\mathbf{w}_{k}$ represents the $N \times 1$ field noise vector. Suppose the modeling noise is smaller than the field noise, we make the following assumption for the properties of $\mathbf{w}_{k}$ and $\mathbf{v}_{k}$.
Assumption 4.1: $\mathbf{v}_{k}$ and $\mathbf{w}_{k}$ are zero mean i.i.d Gaussian with covariance matrices $V_{k}=E\left[\mathbf{v}_{k} \mathbf{v}_{k}^{T}\right]=\sigma_{1}^{2} I_{3 \times 3}$ and $W_{k}=$ $E\left[\mathbf{w}_{k} \mathbf{w}_{k}^{T}\right]=\sigma_{2}^{2} I_{3 \times 3}$, in which $\sigma_{1}<\sigma_{2}$.

Based on the state and measurement equations, the cooperative Kalman filter can be computed. For details about computing the cooperative Kalman filer, readers can refer to [8]. If we define the error covariance matrix of the cooperative Kalman filter at time instant $t_{k}$ as $P_{k}$, then, $P_{k}$ satisfies $P_{k}^{-1}=\left[A_{k} P_{k} A_{k}^{T}+V_{k}\right]^{-1}+C_{k}^{T} W_{k}^{-1} C_{k}$.

Suppose we control the agents in the group to form a symmetric formation. The distance from each agent to the center of the formation is $a$, which means $\left\|\mathbf{x}_{i, k}-\mathbf{x}_{c, k}\right\|=a$. As $k \rightarrow \infty, A_{k}$ goes to $I_{3 \times 3}$ since $\left(\mathbf{x}_{c, k}-\mathbf{x}_{c, k-1}\right) \rightarrow 0$. For simplicity, we drop the subscript $\infty$ in the following derivations. We calculate that, as $k \rightarrow \infty, \quad C^{T} W^{-1} C=\frac{1}{\sigma_{2}^{2}} \operatorname{diag}\left(N, \frac{1}{2} a^{2} N, \frac{1}{2} a^{2} N\right)$ holds [19], [25]. Hence, we obtain $P$ as $P=\operatorname{diag}\left(-\frac{1}{2} \sigma_{1}^{2}+\right.$ $\frac{\sigma_{1}}{2} \sqrt{\sigma_{1}^{2}+\frac{4 \sigma_{2}^{2}}{N}},-\frac{1}{2} \sigma_{1}^{2}+\frac{\sigma_{1}}{2} \sqrt{\sigma_{1}^{2}+\frac{8 \sigma_{2}^{2}}{a^{2} N}},-\frac{1}{2} \sigma_{1}^{2}+$ $\left.\frac{\sigma_{1}}{2} \sqrt{\sigma_{1}^{2}+\frac{8 \sigma_{2}^{2}}{a^{2} N}}\right)=\operatorname{diag}(P(1), P(2), P(3))$, where $P(1)$ is the error covariance of the filtered measurements and $P(2)$ and $P(3)$ correspond to the error covariance of the field gradient estimation at the center of the formation. Note that we use $\sigma_{2}^{2}$ to represent the field noise variance in cooperative exploration. To evaluate the noise reduction ability, we need to compare $P(1)$ with $\sigma_{2}^{2}$ by using multiple agents. We provide the following lemma to address this comparison.

Lemma 4.2: Consider that $N$ sensing agents move in field $Y(\mathbf{x})$ in a symmetric formation based on the cooperative exploration strategy introduced in [8]. Under Assumption 4.1 , the error variance of the filtered measurements $P(1)$ is less than the variance of field noise $\sigma_{2}$ if $N \geq 2$.

Proof: According to Assumption 4.1, since $\sigma_{1}<\sigma_{2}$, we write $\sigma_{1}=\frac{1}{m} \sigma_{2}$, in which $m>1$ is a constant. Substituting $\sigma_{1}$ into $P(1)$ yields $P(1)=\frac{1}{2 m^{2}} \sqrt{1+\frac{4}{N}} \sigma_{2}^{2}$. If we set $P(1)<\sigma_{2}^{2}$, then $N>\frac{4}{4 m^{4}-1}$. Since $m>1$, which indicates $\frac{4}{4 m^{4}-1}<\frac{4}{3}$, then as long as $N>\frac{4}{3}, P(1)<\sigma_{2}^{2}$ holds. Therefore, since $N$ should be an integer, $N \geq 2$ guarantees that cooperative exploration reduces the field noise.

The expression of $P(1)$ indicates that as the number of agents $N$ increases, $P(1)$ decreases. Therefore, by relating $P(1)$ to $\sigma_{2}^{2}$, we can evaluate the lower bound of the required number of agents to guarantee coherent steps of the superagent. To state the relationships, we propose the following proposition.

Proposition 4.3: Consider field $Y(\mathbf{x})=F(\mathbf{x})+W(\mathbf{x})$, where $\varepsilon>0$ and $0<p<1$ are two constants. Under Assumption 4.1, suppose a super-agent of $N$ collaborating agents in a symmetric formation moves one step from $\mathbf{x}_{c, 0}$ to $\mathbf{x}_{c} \in \Omega$ with step size $\delta_{1}$ in the field. Assume that the following conditions are satisfied by the step.

(1) If $\mathbf{x}_{c} \in U^{+}(\varepsilon) \cap B\left(\delta_{1}\right)$ or $\mathbf{x}_{c} \in U^{-}(\varepsilon) \cap B\left(\delta_{1}\right)$, $N$ satisfies

$$
N>\frac{16 \sigma_{2}^{4}}{m^{2}\left(\frac{\left|F\left(\mathbf{x}_{c}\right)-F\left(\mathbf{x}_{c, 0}\right)\right|-\varepsilon}{e r f^{-1}(p)}\right)^{4}-4 \sigma_{2}^{4}} .
$$


(2) If $\mathbf{x}_{c} \in U^{0}(\varepsilon) \cap B\left(\delta_{1}\right), N$ satisfies

$$
\begin{gathered}
\operatorname{erf}\left(\frac{\sqrt{2} m\left(\varepsilon-\left(F\left(\mathbf{x}_{c}\right)-F\left(\mathbf{x}_{c, 0}\right)\right)\right)}{2\left(1+\frac{4}{N}\right)^{\frac{1}{4}} \sigma_{2}}\right)+\operatorname{erf}\left(\frac{\sqrt{2} m\left(\varepsilon+\left(F\left(\mathbf{x}_{c}\right)-F\left(\mathbf{x}_{c, 0}\right)\right)\right)}{2\left(1+\frac{4}{N}\right)^{\frac{1}{4}} \sigma_{2}}\right) \\
>1+p,
\end{gathered}
$$

then, the step is coherent.

Proof: Substituting $P(1)^{\frac{1}{2}}=\frac{1}{\sqrt{2} m}\left(1+\frac{4}{N}\right)^{\frac{1}{4}} \sigma_{2}$ for $\sigma$ in Equations (2) and (3) yields Equations (7) and (8). According to the definition of coherent steps, if $N$ satisfies Equations (7) and (8), the step is coherent.

Based on the Proposition 4.3, if a step of an agent from location $\mathbf{x}_{0}$ to $\mathbf{x}$ is incoherent, we can increase the number of agents in the field and let them perform cooperative exploration. If the number of agents $N$ satisfies Equations (7) and (8), then the step of the super-agent in cooperative exploration is coherent, which indicates that the probability of false-walks of the super-agent can be guaranteed to be less than $\frac{1-p}{2}$. However, increasing the number of agents does not work for all steps. For those steps satisfying $F\left(\mathbf{x}_{c}\right)-$ $F\left(\mathbf{x}_{c, 0}\right)= \pm \varepsilon$, Equation (8) becomes $\operatorname{erf}\left(\frac{\sqrt{2} m \varepsilon)}{\left(1+\frac{4}{N}\right)^{\frac{1}{4}} \sigma_{2}}\right)>1+p$, which is impossible since $\operatorname{erf}(\cdot) \leq 1$. In this case, the step is incoherent regardless of the number of sensing agents $N$.

\section{ApPlications and Experimental Results}

\section{A. Estimating the minimum number of agents}

In this section, we design a strategy for estimating the required minimum number of agents that guarantees coherent steps in a task of seeking a local minimum of a field. We allow the agents to self-organize into groups with an estimated minimum number of agents so that the rest of the agents can be saved for other exploration tasks.

Suppose we deploy $N$ sensing agents in field $Y(\mathbf{x})$. We assume that there are strategies that allow the sensing agents to estimate the moving directions in the field so that measurements can be reduced along the trajectories of the agents.

Suppose all the agents move individually in the field while estimating the field gradients for a certain number of steps. We provide the following algorithm that the agents can use to estimate the required minimum number of agents and start cooperative exploration.

Algorithm 5.1: Given field $Y(\mathbf{x})=F(\mathbf{x})+W(\mathbf{x})$ and $\varepsilon>0$ and $0<p<1$, suppose $N$ agents are deployed in the field.

S.1 From time instant $k=1$, the agents individually perfor$\mathrm{m}$ gradient descending according to any strategy that reduces sensor readings.

S.2 At $k=M$, the $i$ th agent estimates the field noise variance by

$$
\hat{\sigma}_{2}^{2}=\frac{1}{M} \sum_{j=1}^{M}\left(y\left(\mathbf{x}_{i, j}\right)-\frac{1}{M} \sum_{l=1}^{M} y\left(\mathbf{x}_{i, l}\right)\right)^{2}
$$

and estimates the number of the agents required to guarantee coherent steps by

$$
N_{\min }>\frac{16 \hat{\sigma}_{2}{ }^{4}}{m^{2}\left(\frac{\left(\left|\delta_{1} \mathbf{h}^{T} \nabla \hat{F}\left(\mathbf{x}_{i, M-1}\right)\right|-\varepsilon\right)}{\operatorname{erf}^{-1}(p)}\right)^{4}-4 \hat{\sigma}_{2}^{4}},
$$

where $\mathbf{h}=\frac{\mathbf{x}_{i, M}-\mathbf{x}_{i, M-1}}{\left|\mathbf{x}_{i, M}-\mathbf{x}_{i, M-1}\right|}$ and $\nabla \hat{F}\left(\mathbf{x}_{i, M-1}\right)$ is the estimated gradient at $\mathbf{x}_{i, M-1}$. Then the $i$ th agent forms a group of $N_{\min }$ with the $N_{\min }-1$ agents closest to it. The distance between the $i$ th and $j$ th agents is measured by $d_{i, j}=$ $\left|\mathbf{x}_{i, k}-\mathbf{x}_{j, k}\right|$. If $N<N_{\min }$, all the agents form a group with $N$ agents.

S.3 From $k=M+1$, the group performs cooperative exploration. A cooperative Kalman filter that is introduced in Section IV is constructed. The center of the formation is directed according to $\dot{\mathbf{x}}_{c, k}=-\nabla F\left(\mathbf{x}_{c, k}\right)$, where $\nabla F\left(\mathbf{x}_{c, k}\right)$ is the output of the cooperative Kalman filter. The remaining agents continue individual exploration.

\section{B. Experimental Results}

To study the performance of the self-organizing algorithm, we deploy five Khepera III robots in a test-bed developed in our lab. The test-bed consists of a standard $40 \mathrm{~W}$ incandescent light bulb that generates a light field, an overhead localization system programmed in LabView, and a central computer. Each Khepera III robot measures the light intensity using the infrared sensors around it and seeks the light source in the light field. For details of the experimental settings, readers can refer to [26].

In the experiments, all the robots start from moving in the field individually. In the first trial, we set $p=0.9$, $\varepsilon=50$, and $m=1.5$, and choose a step size of $\delta_{1}=5 \mathrm{~cm}$ for the robots. Following Algorithm 5.1, at step $k=8$, robot "G" first requires collaboration. For the robots to decide whether they need collaboration, we use the switching conditions introduced in our paper [26]. Robot "G" estimates $\sigma_{2}^{2}$ and $N_{\min }$ according to Equations (9) and (10) and obtains $\sigma_{2}=341.0085$ and $N_{\min }>2.8429$. Therefore, $N_{\min }=3$. The closest two robots to Robot "G", robots "O" and "N", together with Robot " $G$ " form a symmetric formation and converge to the light source. Since the estimation results indicate that three robots are enough to guarantee coherent steps with a probability of $90 \%$ in the field, the left two robots perform individual exploration all the time. We can also assign other tasks to the remaining two robots. Fig. 1 (left) shows the trajectories of the five robots. If we increase the desired explorable probability $p$ to 0.91 , the estimated minimum number becomes $N_{\min }=4$. The trajectories of the four robots are illustrated in Fig. 1 (right). Fig. 2 shows the measurements collected by the agents in these two cases. The blue line with triangles are the filtered field values at the center of the formation, and the vertical dark blue lines indicate the time step, at which switching occurs.

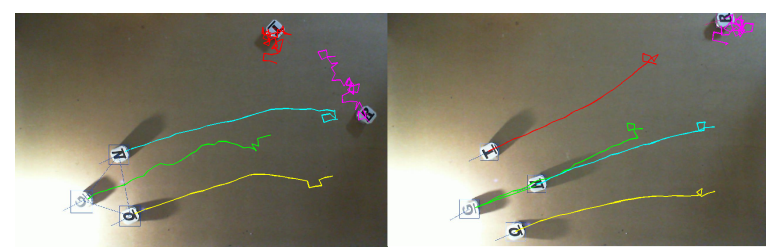

Fig. 1. Trajectories of five Khepera III robots. $N_{\min }=3$ (left), and $N_{\min }=4$ (right). 

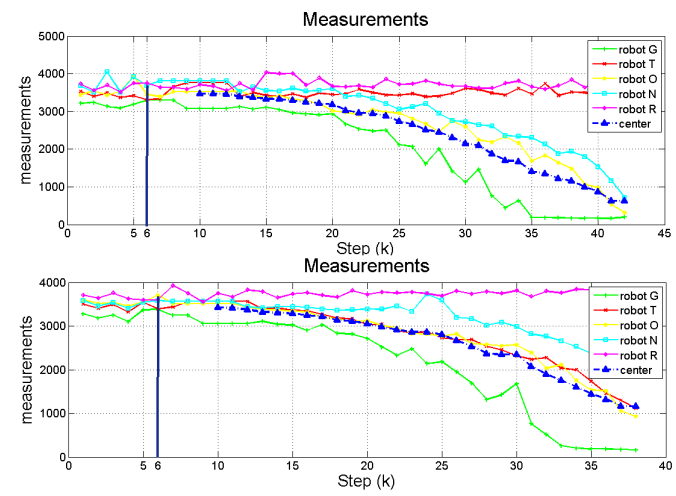

Fig. 2. Measurements of five Khepera III robots in the first $\left(N_{\min }=3\right)$ and second $\left(N_{\min }=4\right)$ experiment.

As we discussed in Section IV, as the number of agents $N$ increases, the error in the cooperative Kalman filter decreases, as shown in Fig. 3, in which the blue, green, and red lines correspond to the traces of the error covariance matrix of the cooperative Kalman filter when three, four, and five robots are in a group, respectively.

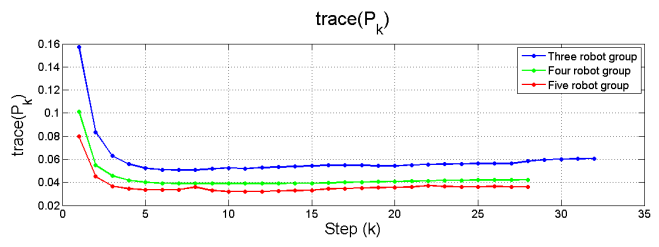

Fig. 3. Traces of the cooperative Kalman filter when three, four, and five robots are performing cooperative exploration.

\section{CONCLUSiOnS AND Future Work}

In this paper, we examine the behaviors of sensing agents in the exploration of unknown noisy scalar fields. We propose notions of coherent steps and incoherent steps of sensing agents and show that a step of an agent being coherent is the sufficient and necessary condition for the probability of a false-walk being less than a certain threshold. We apply the cooperative exploration strategy and prove that as the number of collaborating agents increases, the steps of the agent group become coherent. We provide an algorithm that estimates the minimum required number of agents that guarantee coherent steps and implement the algorithm in experiments. Future work includes applying the explorability analysis in nonGaussian fields and implementing the proposed algorithm in real-world applications.

\section{REFERENCES}

[1] P. Ogren, E. Fiorelli, and N. E. Leonard, "Cooperative control of mobile sensor networks: Adaptive gradient climbing in a distributed environment," IEEE Transactions on Automatic Control, vol. 49, no. 8, pp. 1292-1302, 2004.

[2] J. Cortes, "Distributed gradient ascent of random fields by robotic sensor networks," in Proc. 46th IEEE Conf. on Decision and Control, 2007, pp. 3120-3126.

[3] B. J. Moore and C. C. de Wit, "Source seeking via collaborative measurements by a circular formation of agents," in Proc. of 2010 American Control Conference, 2010, pp. 6417-6422.
[4] S. Azuma, M. Sakar, and G. Pappas, "Stochastic source seeking by mobile robots," IEEE Transactions on Automatic Control, no. 99, 2012, early Access.

[5] E. Biyik and M. Arcak., "Gradient climbing in formation via extremum seeking and passivity-based coordination rules," in Proc. of 46th IEEE Conf. on Decision and Control, 2007, pp. 3133-3138.

[6] M. Kemp, A. L. Bertozzi, and D. Marthaler, "Multi-UUV perimeter surveillance," in Proc. of the 2004 IEEE/OES Workshop on Autonomous Underwater Vehicles, 2004, pp. 102-107.

[7] S. Susca, S. Martínez, and F. Bullo, "Monitoring environmental boundaries with a robotic sensor network," IEEE Transactions on Control Systems Technology, vol. 16, no. 2, pp. 288-296, 2008.

[8] F. Zhang and N. E. Leonard, "Cooperative control and filtering for cooperative exploration," IEEE Transactions on Automatic Control, vol. 55 , no. 3, pp. 650-663, 2010.

[9] W. Wu and F. Zhang, "Cooperative exploration of level surfaces of three dimentional scalar fields," Automatica, vol. 47(9), pp. 20442051, 2011.

[10] E. Burian, D. Yoerger, A. Bradley, and H. Singh, "Gradient search with autonomous underwater vehicles using scalar measurements," in Proceedings of the 1996 Symposium on Autonomous Underwater Vehicle Technology, 1996, pp. 86 - 98.

[11] C. Zhang, D. Arnold, N. Ghods, A. Siranosian, and M. Krstic, "Source seeking with nonholonomic unicycle without position measurement and with tuning of forward velocity," Systems and Control Letters, vol. 56, pp. 245-252, 2007.

[12] C. G. Mayhew, R. G. Sanfelice, and A. R. Teel, "Robust hybrid source-seeking algorithms based on directional derivatives and their approximations," in Proc. of 47th IEEE Conf. Decision and Control, 2008, pp. $1735-1740$.

[13] J. Cochran and M. Krstic, "Nonholonomic source seeking with tuning of angular velocity," IEEE Transactions on Automatic Control, vol. 54, no. 4, pp. 717-731, 2009.

[14] W. H. Moase, C. Manzie, and M. J. Brear, "Newton-like extremumseeking part I: Theory," in Proc. of 48th IEEE Conf. on Decision and Control, 2009, pp. 3839-3844.

[15] A. Jadbabaie, J. Lin, and A. Morse, "Coordination of groups of mobile autonomous agents using nearest neighbor rules," IEEE Transactions on Automatic Control, vol. 48, no. 6, pp. 988-1001, 2003.

[16] W. Burgard, M. Moors, C. Stachniss, and F. E. Schneider, "Coordinated multi-robot exploration," IEEE Transactions on Robotics, vol. 21, no. 3, pp. 376-386, 2005.

[17] B. Grocholsky, J. Keller, V. Kumar, and G. Pappas, "Cooperative air and ground surveillance," IEEE Robotics and Automation Magazine, vol. 13 , no. 3, pp. 16-25, 2006.

[18] W. Ren, R. Beard, and E. Atkins, "Information consensus in multivehicle cooperative control," Control Systems, IEEE, vol. 27, no. 2, pp. 71-82, 2007.

[19] W. Wu and F. Zhang, "Explorability of noisy scalar fields," in Proc. 2011 IEEE Conference on Decision and Control, 2011, pp. 64396444.

[20] M. Dorigo, V. Trianni, E. Sahin, R. Gro, T. Labella, G. Baldassarre, S. Nolfi, J.-L. Deneubourg, F. Mondada, D. Floreano, and L. Gambardella, "Evolving self-organizing behaviors for a swarm-bot," Autonomous Robot, vol. 17, no. 2-3, pp. 223-245, 2004.

[21] J. Halloy, G. Sempo, G. Caprari, C. Rivault, M. Asadpour, F. Tche, I. Sad, V. Durier, S. Canonge, J. M. Am, C. D. N. Correll, A. Martinoli, F. Mondada, R. Siegwart, and J. L. Deneubourg1, "Social integration of robots into groups of cockroaches to control self-organized choices," Science, vol. 318, no. 5853, pp. 1155-1158, 2007.

[22] S. Nouyan, R. Gross, M. Bonani, F. Mondada, and M. Dorigo, "Teamwork in self-organized robot colonies," IEEE Transactions on Evolutionary Computation, vol. 13, no. 4, pp. 695-711, 2009.

[23] F. Zhang, "Geometric cooperative control of particle formations," IEEE Transactions on Automatic Control, vol. 55, no. 3, pp. 800-803, 2010.

[24] H. Yang and F. Zhang, "Robust control of formation dynamics for autonomous underwater vehicles in horizontal plane," ASME Journal of Dynamic Systems, Measurement and Control, vol. 134, no. 3, p. 031009, 2012.

[25] W. Wu and F. Zhang, "Experimental validation of source seeking with a switching strategy," in Proc. 2011 IEEE Conference on Robotics and Automation, 2011, pp. 3835-3840.

[26] — - "Robust cooperative exploration with a switching strategy," IEEE Transactions on Robotics, p. accepted, 2012. 